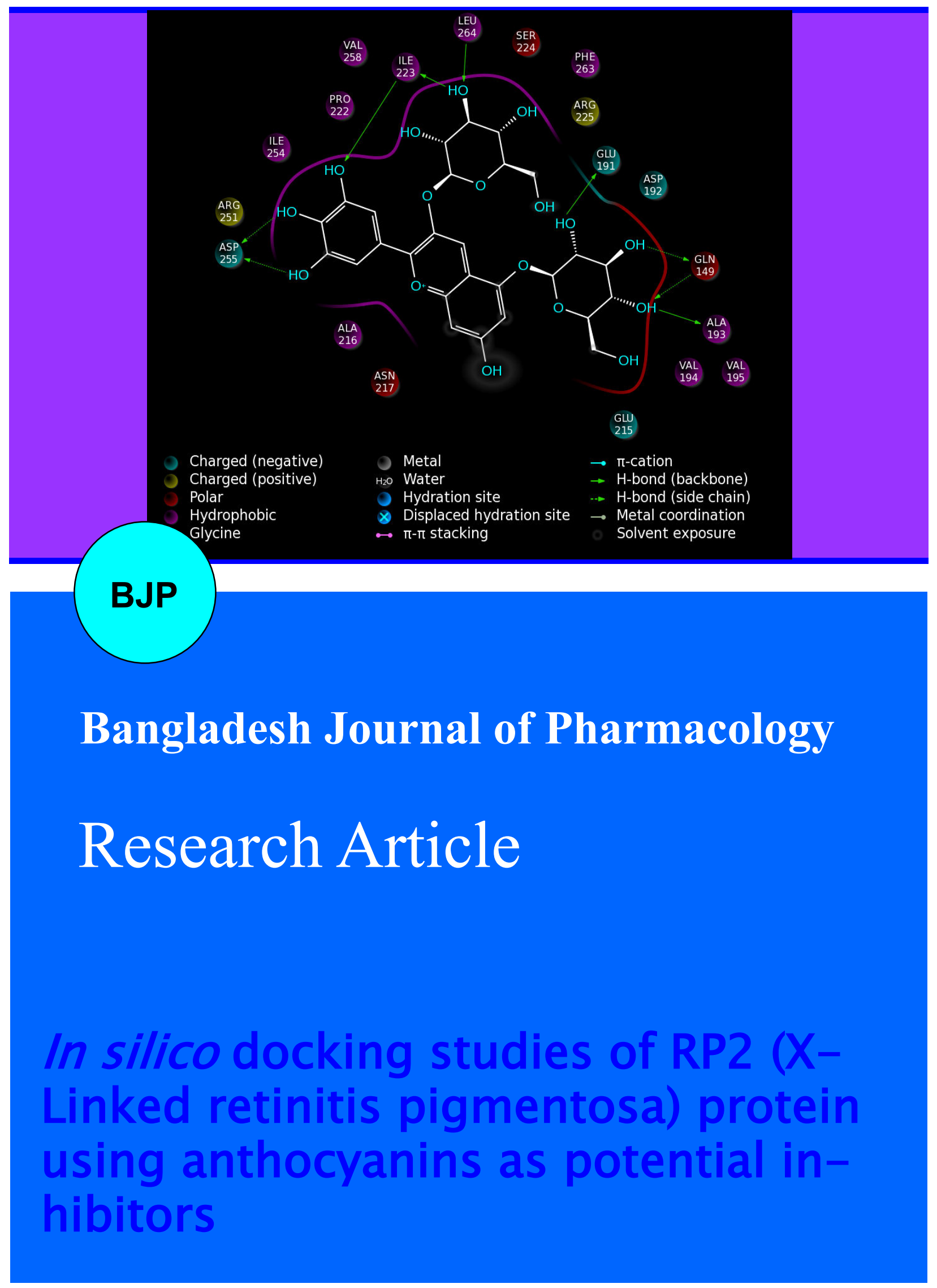




\title{
In silico docking studies of RP2 (X-Linked retinitis pigmentosa) protein using anthocyanins as potential inhibitors
}

\author{
Sathyan Sri Lavvanya Priya', Ponnusamy Renuka Devi ${ }^{1}$ and Arumugam Madeswaran ${ }^{2}$ \\ ${ }^{1}$ Department of Biotechnology, Anna University, Regional Center, Coimbatore, Jothipuram, Coimbatore 641047 , \\ Tamil Nadu, India; ${ }^{2}$ Department of Pharmacology, College of Pharmacy, Sri Ramakrishna Institute of Paramedical \\ Sciences, 395, Sarojini Naidu Road, Siddhapudur, Coimbatore 641 044, India.
}

\begin{tabular}{|c|c|}
\hline \multicolumn{2}{|l|}{ Article Info } \\
\hline Received: & 28 May 2013 \\
\hline Accepted: & 6 June 2013 \\
\hline Available Online: & 30 June 2013 \\
\hline \multicolumn{2}{|c|}{ DOI: 10.3329/bjp.v8i3.15110 } \\
\hline \multicolumn{2}{|c|}{$\begin{array}{l}\text { Cite this article: } \\
\text { Priya SSL, Devi PR, Madeswaran A. } \\
\text { In silico docking studies of RP2 (X- } \\
\text { Linked retinitis pigmentosa) protein } \\
\text { using anthocyanins as potential inhib- } \\
\text { itors. Bangladesh J Pharmacol. 2013; 8: } \\
\text { 292-99. }\end{array}$} \\
\hline
\end{tabular}

\begin{abstract}
Mutations occurring in X-Linked retinitis pigmentosa (RP2) gene causes retinal damage, ultimately leading to vision loss. Owing to the complications of the disease, there is no effective treatment till date. In the present study, we have refined the crystal structure of RP2 using protein preparation wizard of Glide software. Active site of the protein was predicted with SiteMap program. Five anthocyanin compounds from Syzygium cumini fruit peel were docked into the active site of RP2. Molecular docking calculations were performed by employing Glide XP algorithm. Cyanidin 3,5 diglucoside with lowest Gscore $(-12.6 \mathrm{kcal} / \mathrm{mol})$ was rescored subsequently with PrimeMMGBSA binding free energy calculations. Binding affinity $(-93.6 \mathrm{kcal} / \mathrm{mol})$ of the inhibitor suggests its potential use in the treatment of retinitis pigmentosa.
\end{abstract}

\section{Introduction}

Vision loss due to age related macular degeneration is common worldwide. Retinitis pigmentosa (RP) is grouped under the inherited diseases category affecting people of age group 20-60. Photoreceptor (rods) layer of the retina is damaged resulting in gradual decrease in vision leading to complete blindness. Appearance of black spots in the retina, loss of peripheral vision (Rundquist, 2004) are few prominent signs of the disease. Two million people are affected (Berson et al., 2012) by RP around the globe. It has been estimated that one in 930 persons and one in 1000 people (Carmen and Jose, 2010) have severe visual impairment caused by RP. Being an inherited disease, RP is autosomally dominant, recessive and X-linked (Inglehearn, 1998). Several genes are involved in causing retinal degenration, making the RP more complicated. The symptoms differ among the patients.

The X-linked recessive pattern is considered the most serious form of RP. The gene encoding RP, termed RP2 (TBCCD2) is localized in the cytoplasm of the cell membrane. Mutations occurring in X-linked RP2 (Hardcastle et al., 1999) is responsible for the progressive vision loss mostly in males (recessive) and sometimes equally affecting both the sexes (dominant). To this date no effective treatment is available for the disease condition. RP2 could be a possible potential drug target (Veltel and Wittinghofer, 2009) for treating $\mathrm{RP}$. Use of valproic acid as drug for RP is limited owing to the side effects (Bhalla et al., 2013). Few studies have suggested the use of Vitamin A supplements ( $\mathrm{Li}$ et al., 1998), and omega-3 fatty acids to slow down the progression of the disease.

Nature provides abundant sources for developing herbal drug leads. The secondary metabolites produced in plants are termed as "bioactive compounds" owing to their pharmacological effects. Past few years there has been a resurgence of interest in characterization of these compounds. Different parts of various plants have 
been studied extensively for their therapeutic applications. Tannins, glycosides, saponins, flavonoids, sequetrepenoids, phytosterols, lignans, alkaloids, proteins and peptides are a broad range of compounds harboured in medicinal plants. These compounds of natural origin that include plants, microbes, and animals form the base for drug discovery (Ji et al., 2009).

Among the bioactive compounds, phenolics are important due to their high anti-oxidant activity. Anthocyanins, belonging to the group of flavonoids, have been reported to exert positive effects on human health (Wrolstad, 2004; Adisakwattana et al., 2011). Many fruits and vegetables rich in anthocyanins are used in combating chronic diseases such as cancer, cardiovascular diseases (Wang and stoner, 2008; Wallace, 2011). Berries from Vaccinium myrtillus are beneficial in enhancing vision (Matsumoto et al., 2001) which is attributed to the significant anti-oxidant potential of the anthocyanins (Ghosh and Konishi, 2007). In silico approaches are widely used to identify the lead molecules for several disorders. In this context, we proposed the efficacy of anthocyanins isolated from Syzygium cumini fruit peel as potential inhibitors for Best's disease (Priya et al., 2012). Therefore in the present study we performed docking studies for the RP2 protein, a potential drug target, using the three anthocyanin compounds we previously identified (cyanidin, malvidin, petunidin). Additionally, two other anthocyanins (peonidin, delphinidin) and valproic acid were docked with the target for purpose of standardization.

\section{Materials and Methods}

\section{Structure retrieval}

The crystal structure of the human retinitis pigmentosa protein 2 (RP2) at $2.1 \AA$ was retrieved from the PDB, (PDB ID: 2BX6) (Kuhnel et al., 2006), consisting of 350 amino acids.

\section{Preparation of target protein}

Using protein preparation wizard of Glide (Schrödinger suite version 9.3) running on red hat enterprise linux 5 (RHEL 5) workstation, the protein was prepared. Firstly, water molecules were removed from the crystallographic structure followed by addition of hydrogen atoms. All atom charges and atom types were assigned. Finally, energy minimization and refinement of the structure was done up to $0.3 \AA$ RMSD by applying OPLS-2005 force field. The optimized target protein was employed for docking studies.

\section{Preparation of ligands}

The ligand files were acquired from the NCBI pubchem database that include cyanidin 3,5 diglucoside (CID 441688), delphinidin 3,5 glucoside (CID 10100906), malvidin 3,5 diglucoside (CID 441765), petunidin 3,7diglucoside (CID 44256973), peonidin 3,5-diglucoside (CID 44256843) and valproic acid (CID 3121) Ligprep protocol (Ligprerp, 2005) was used for minimization of the ligands (Figure 1). The default parameters included: Ionizers, generating tautomers, generating possible conformers at $\mathrm{pH} 7$ with OPLS_2005.1 force filed

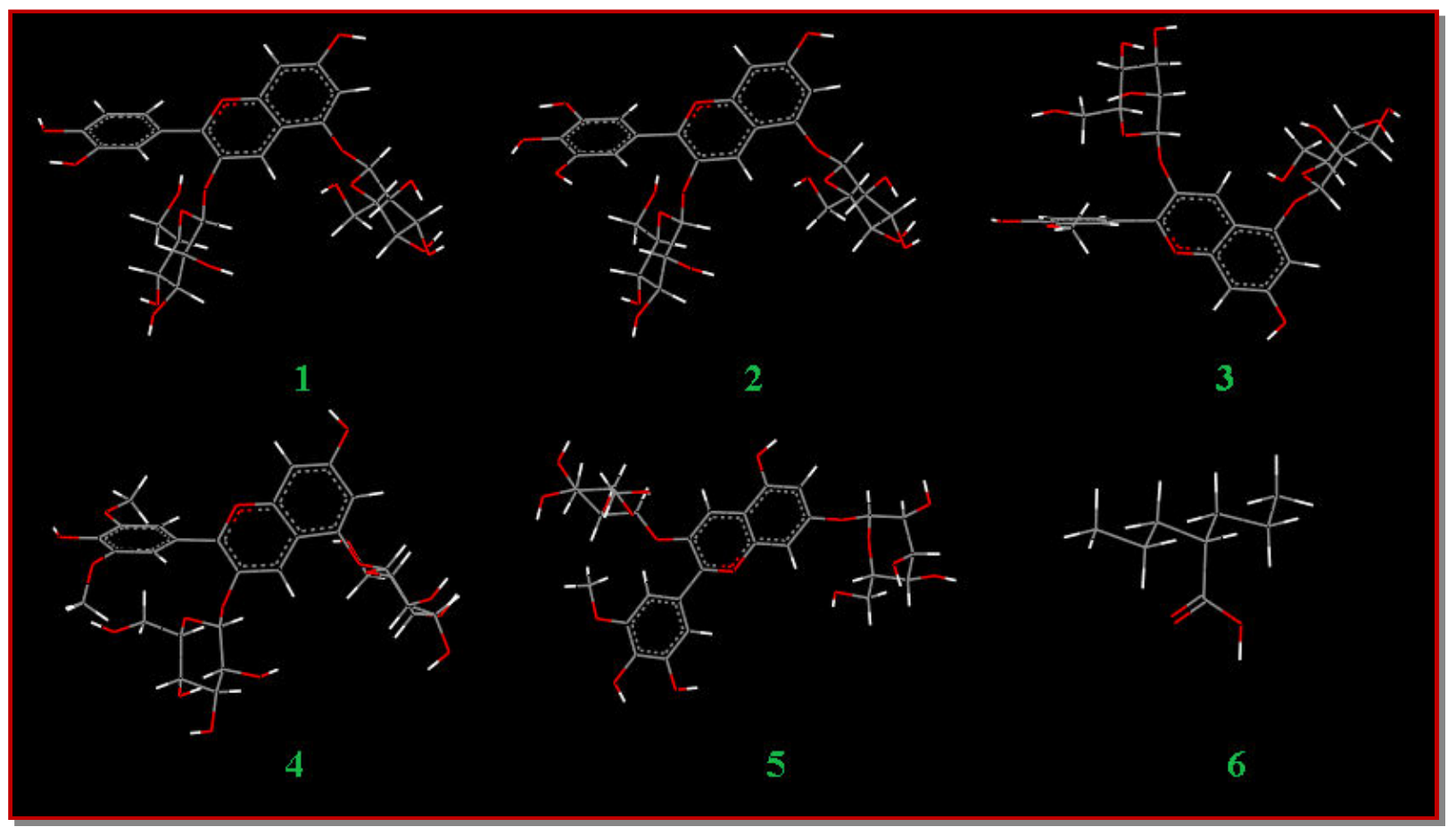

Figure 1: The optimized ligand molecules (1 Cyanidin 3,5 diglucoside, 2 Delphinidin 3,5 diglucoside, 3 Peonidin 3,5 diglucoside, 4 Malvidin 3,5 diglucoside, 5 Petunidin 3,5 diglucoside, 6 Valproic acid) 


\begin{tabular}{|c|c|c|c|c|c|c|c|c|c|c|}
\hline \multicolumn{11}{|c|}{ Table I } \\
\hline \multicolumn{11}{|c|}{ Active site prediction using SiteMap } \\
\hline Sitemap & Site score & Size & Volume & Exposure & Enclosure & Contact & Phobic & Philic & Balance & Don/acc \\
\hline Site 1 & 0.988305 & 154 & 300.1 & 0.540299 & 0.680511 & 0.942128 & 0.262247 & 1.351018 & 0.194110 & 0.576586 \\
\hline Site 2 & 0.807282 & 58 & 166.7 & 0.681319 & 0.602465 & 0.755268 & 0.492843 & 0.802173 & 0.614385 & 1.237848 \\
\hline Site 3 & 0.805873 & 60 & 194.5 & 0.700000 & 0.654842 & 0.852080 & 0.110590 & 1.130570 & 0.097818 & 0.792475 \\
\hline Site 4 & 0.750694 & 49 & 97.6 & 0.581197 & 0.654117 & 0.851421 & 0.040010 & 1.242985 & 0.032188 & 0.432637 \\
\hline Site 5 & 0.700358 & 29 & 68.9 & 0.691489 & 0.577005 & 0.839499 & 1.310087 & 0.418575 & 3.129875 & 4.753712 \\
\hline
\end{tabular}

thereby achieving the correct protonated state for each ligand used.

\section{Prediction of active sites}

SiteMap (Halgren, 2009) is a robust program that analyses the characteristic features of binding sites by following steps: A thorough initial search step results in identification of one or more regions on the receptor surface which facilitates binding of the ligand with receptor. Hydrophobic and hydrophilic maps are generated, the latter is further dispersed as donor, acceptor and metal binding regions. Each identified site is assessed by calculation of SiteScore involving physical parameters comprising of volume, site size, exposure/ enclosure hydrophilic, hydrogen bond donor/acceptor isosurfaces, enclosure/exposure are used to characterize the binding pockets. Generally a good SiteScore of a binding site is 1.0 (Lauria et al., 2009). SiteScore, the scoring function, accurately ranks the site with highest score determines the drug-ability. Therefore the binding sites of the RP2 protein were identified using SiteMap.

\section{Receptor grid generation and binding site prediction}

Prior to docking, receptor grid generation is an essential step. Co-crystallized ligand was excluded from the crystallized RP2 protein. Centroid of the residues, predicted by SiteMap was defined as the grid box. Van der waals scaling factor 1.00 , charge partial cutoff 0.25 and OPLS_2005 force filed were default parameters used for grid generation.

\section{Molecular docking studies}

The six ligands prepared by ligprep were docked into the active sites of RP2 target using the "extra-precision" (XP) mode of the Glide docking program (Maestro version 9.3). Improved sampling methods and extended ChemScore (Eldridge et al., 1997) function are the characteristic features of Glide-XP. The protocol facilitates docking by ligand flexibility and generation of multiple conformers within the rigid receptor. Maestro Ligand interaction 2-D diagram was used to understand the interaction between the inhibitors and RP2. Best conformation for each ligand was chosen based on the lowest glide score (Gscore). Ligands that form hydrogen bonds with at least one active site of the target protein with a good binding affinity analyses the final Gscore.

\section{Rescoring using Prime MM-GBSA}

Binding affinity of the ligand with the receptor was further estimated using Prime MM-GBSA method (Prime, version 3.1). The ligand that had the lowest Gscore (cyanidin 3,5 diglu-coside) was rescored. Minimization of the complex was performed using optimization option in Prime. By applying OPLS-AA force field and generalized-Born surface area (GBSA) continuum solvent model, the binding free energy of the docked pose (Pearlman, 2005) was calculated with: $\Delta G_{\text {bind }}=G(P L)-G(P)-G(L), P L=$ protein-ligand complex $\mathrm{P}=$ Protein, $\mathrm{L}=$ Ligand.

\section{Results and Discussion}

The prepared target RP2, subjected to SiteMap analysis yielded five active sites. Based on the sitescore, site 1 was chosen for performing the molecular docking studies (Table I). The active sites predicted by SiteMap are Gln 149, Ala 193, Asp 255, Leu 264, Ile 223 and Glu 191. The prediction of potential active sites plays a major role in structure based drug discovery. SiteMap is one of the reliable and high performance methods to predict the active sites in a protein (Halgren, 2009) and also provides an insight into the inhibitor-target interactions.

In silico docking results of the five anthocyanin inhibitors and valproic acid conducted using Glide-XP protocol is depicted in Table II. The quest for novel compounds as inhibitors for treating diseases is the fundamental of drug discovery and development. However, the time and cost involved in the drug discovery process is cumbersome (Dimasi et al., 2003). Therefore, structure based drug design has gained importance which is facilitated through computational docking studies employing programs such as GLIDE docking. In our present study, the inhibitors were ranked based on the glide XP Gscore, glide energy and glide emodel scores. Among the inhibitors docked around the active site of RP2, cyanidin 3,5 diglucoside displayed a high glide XP score of $-12.6 \mathrm{kcal} / \mathrm{mol}$, glide energy of $-64.3 \mathrm{kcal} / \mathrm{mol}$ and a glide emodel score of $88.3 \mathrm{kcal} / \mathrm{mol}$. Residues - Residues Leu 264, Ile 223, Ser 
Table II

In silico docking results of five anthocyanin inhibitors and valproic acid conducted using Glide-XP protocol

\begin{tabular}{|c|c|c|c|c|c|}
\hline Compound & Glide XP Gscore & $\begin{array}{c}\text { Glide energy } \\
\text { (Kcal/ mol) }\end{array}$ & Glide emodel & Interactions & Distance $(\AA)$ \\
\hline $\begin{array}{l}\text { Cyanidin } 3,5 \\
\text { diglucoside }\end{array}$ & -12.6 & -64.3 & -88.3 & $\begin{array}{c}\text { Leu (264) N-H...O } \\
\text { Gln (149) N-H...O } \\
\text { O-H...O(Asp 255) } \\
\text { O-H...O(Asp 255) } \\
\text { O-H...O(Ile 223) } \\
\text { O-H...O(Ala 193) } \\
\text { O-H...O (Gln149) } \\
\text { Arg (251) N-H...O } \\
\text { O-H...O (Ser 219) }\end{array}$ & $\begin{array}{l}3.2 \\
2.9 \\
2.8 \\
2.7 \\
2.7 \\
2.9 \\
2.6 \\
2.7 \\
2.8\end{array}$ \\
\hline $\begin{array}{l}\text { Delphinidin } 3,5 \\
\text { diglucoside }\end{array}$ & -12.6 & -67.3 & -92.7 & $\begin{array}{c}\text { O-H...O(Ala 193) } \\
\text { O-H...O(Glu 191) } \\
\text { O-H...O(Gln 149) } \\
\text { O-H...O(Ile 223) } \\
\text { (Ile 223)N-H...O } \\
\text { O-H...O(Leu 264) } \\
\text { O-H...O(Asp 255) }\end{array}$ & $\begin{array}{l}2.7 \\
3.0 \\
2.9 \\
2.9 \\
3.2 \\
2.9 \\
2.7\end{array}$ \\
\hline $\begin{array}{l}\text { Peonidin } 3,5 \\
\text { diglucoside }\end{array}$ & -11.7 & -67.7 & -90.7 & $\begin{array}{c}\text { O-H...O(Glu 191) } \\
\text { O-H...O(Asp 255) } \\
\text { O-H...O(Leu 264) } \\
\text { O-H...O(Gln 149) } \\
\text { O-H...O(Ile 223) }\end{array}$ & $\begin{array}{l}2.9 \\
2.6 \\
2.2 \\
3.0 \\
2.9\end{array}$ \\
\hline $\begin{array}{l}\text { Malvidin } 3,5 \mathrm{di} \\
\text { glucoside }\end{array}$ & -8.7 & -60.6 & -70.8 & $\begin{array}{c}\text { O-H...O(g1u 215) } \\
\text { O-H...O(Ala 216) } \\
\text { O-H...O(Asp 255) } \\
\text { O-H...O(Asp 255) } \\
\text { O-H...O(gln 149) } \\
\text { O-H...O(Ile 223) } \\
\text { N-H...O (Leu 264) }\end{array}$ & $\begin{array}{l}3.0 \\
3.0 \\
2.8 \\
2.7 \\
3.0 \\
3.0 \\
2.6\end{array}$ \\
\hline $\begin{array}{l}\text { Petunidin } 3,7 \\
\text { diglucoside }\end{array}$ & -7.5 & -55.8 & -80.8 & $\begin{array}{c}\text { O-H...O(Glu 191) } \\
\text { O-H...O(Asp 255) } \\
\text { O-H...O(Asp 255) } \\
\text { O-H...O(Ile 223) }\end{array}$ & $\begin{array}{l}2.8 \\
2.7 \\
2.7\end{array}$ \\
\hline Valproic acid & -1.7 & -13.8 & -11.9 & (Arg 78) N-H...O & 2.6 \\
\hline
\end{tabular}

\begin{tabular}{|c|c|}
\hline \multicolumn{2}{|c|}{ Table III } \\
\hline \multicolumn{2}{|c|}{$\begin{array}{l}\text { Binding free energy calculations for the top } \\
\text { inhibi-tor using Prime MM-GBSA }\end{array}$} \\
\hline Inhibitor & Cyanidin 3,5 diglucoside \\
\hline MM-GBSA E_COMPLEX & -93.6 \\
\hline MM-GBSA E_COMPLEX & -13810.5 \\
\hline MM-GBSA E_PROTEIN & -13755.7 \\
\hline MM-GBSA E_LIGAND & 38.8 \\
\hline
\end{tabular}

219, Arg 251, Asp 255, Ala 193, Gln 149 were found strongly interacting with the compound. An increased interest in cyanidin 3,5 diglucoside is due to its promising health benefits related to vision (Matsunaga et al., 2009). Delphinidin 3,5 diglucoside generated a glide XP score of $-12.6 \mathrm{kcal} / \mathrm{mol}$, with a glide energy and glide emodel score of -67.3 and $-92.7 \mathrm{kcal} / \mathrm{mol}$ respectively. The glide XP Gscores of peonidin 3,5 diglucoside, malvidin 3,5 diglucoside and petunidin 3,7 diglucoside were $-11.7,-8.7,-7.5 \mathrm{kcal} / \mathrm{mol}$ and the glide energies, glide emodel scores were moderate. However, the five inhibitors we docked with the target RP2, formed hydrogen bonds with all the active site residues (Figures 2-6).

Valproic acid interacted with a Arg 78, a non-active site residue forming one hydrogen bond. The glide XP Gscore, glide energy and glide emodel scores (-1.7, -13.8 and $-11.9 \mathrm{kcal} / \mathrm{mol}$ ) were comparatively very less when compared with the natural anthocyanin compounds we docked (Figure 7). The insignificant scores exhibited by valproic acid indicate that it is an unsuitable inhibitor for X-linked retinitis pigmentosa. Possible therapeutic effects of several fruits including berries accounts to the high content of flavonoids, in particular anthocyanins, have been well documented by researchers throughout the world (Kong et al., 2003; Byamukama et al., 2005).

The docking score of the anthocyanins were further improved using the rescoring performed by Prime MMGBSA binding free energy calculations $(\Delta G)$. Results of 


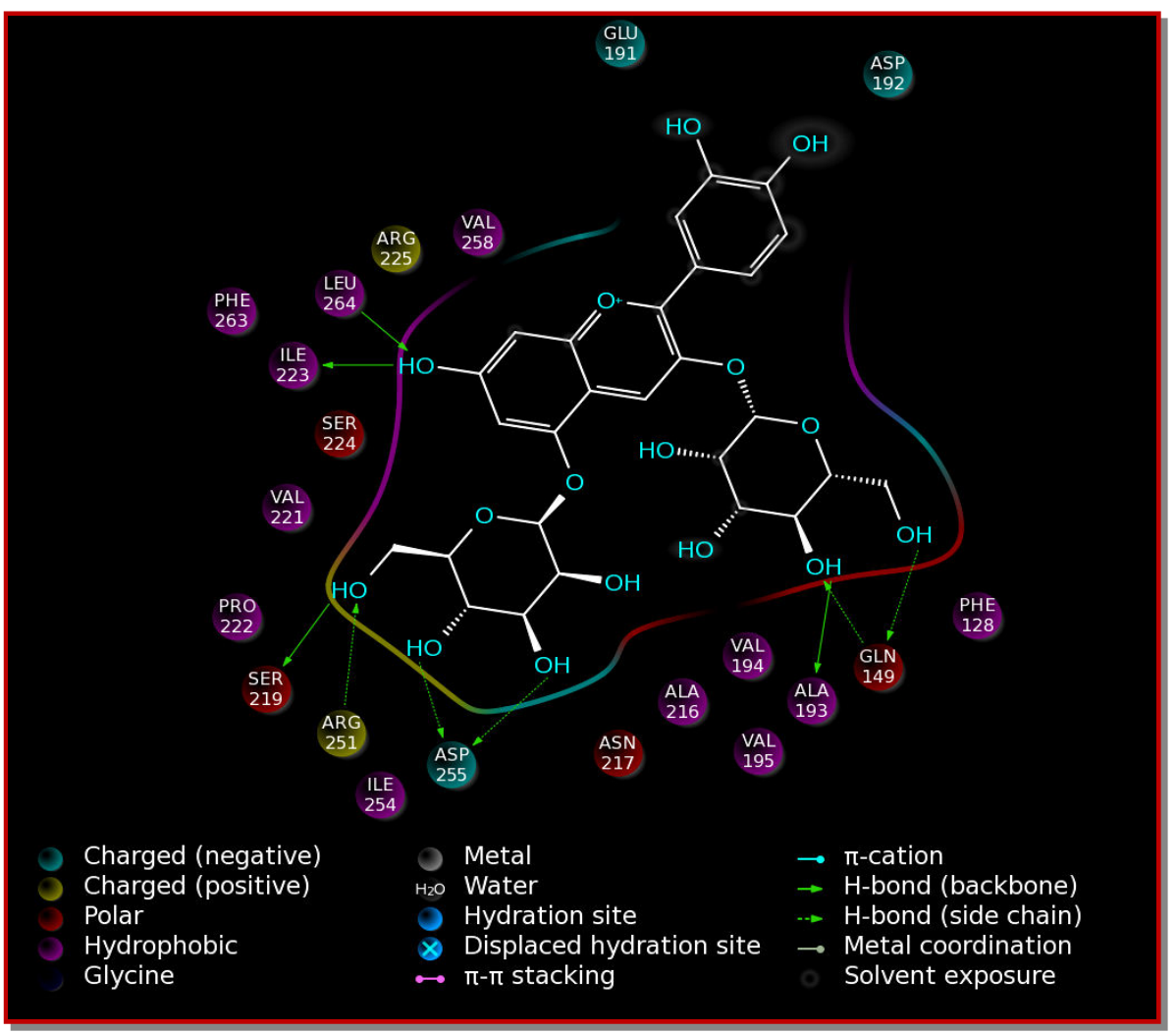

Figure 2: Interaction diagram of cyanidin 3,5 diglucoside

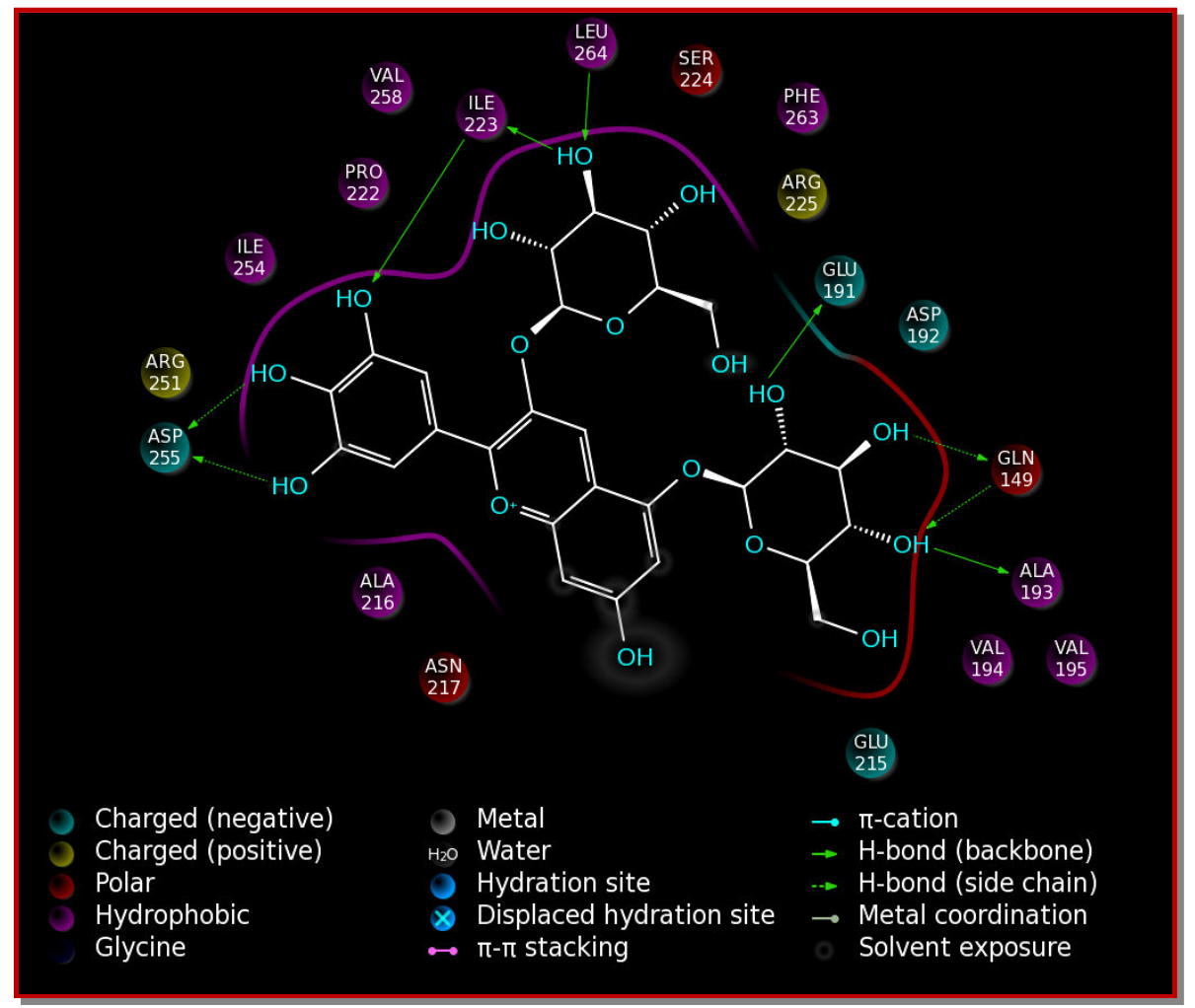

Figure 3: Interaction diagram of delphinidin 3,5 diglucoside 


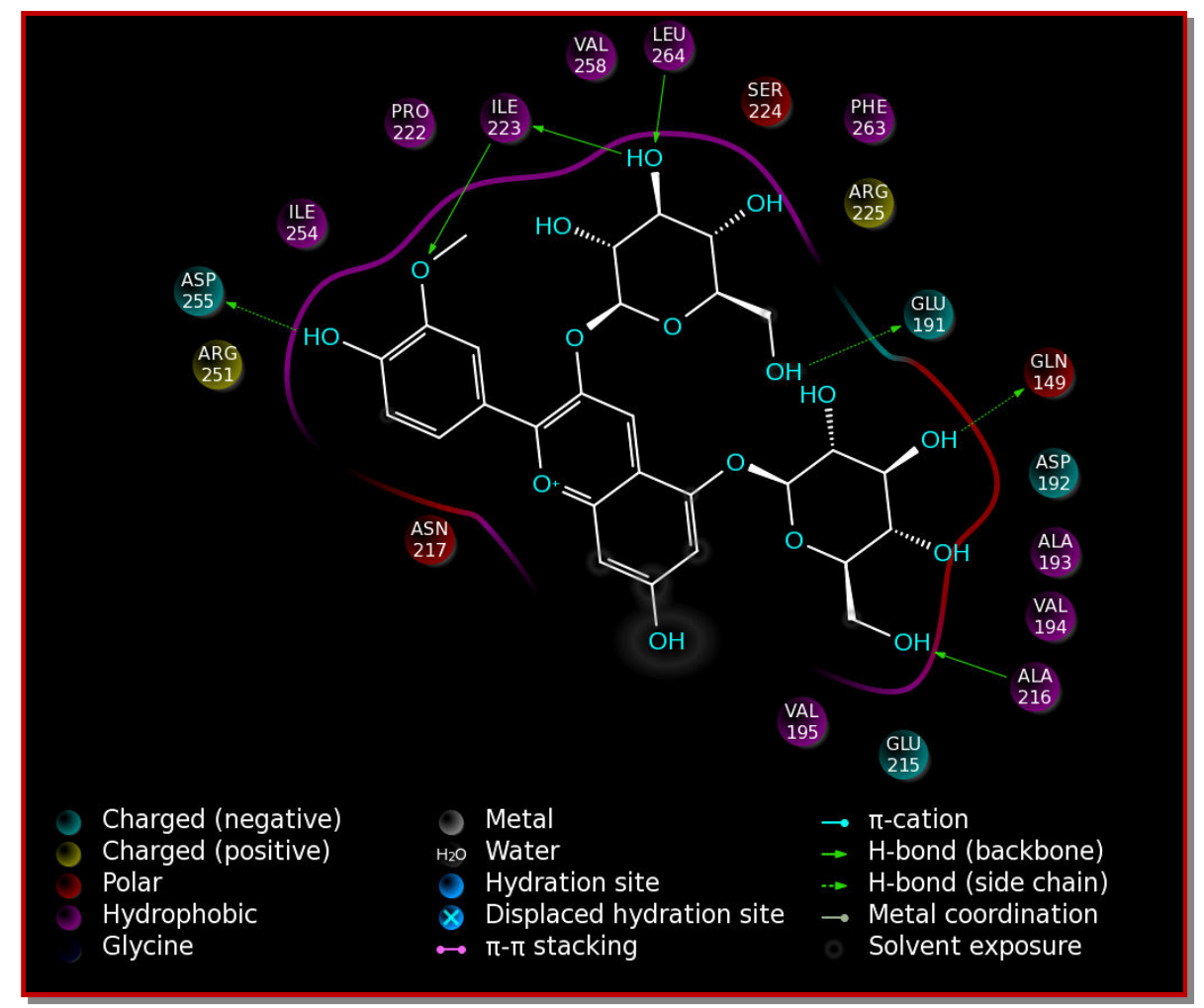

Figure 4: Interaction diagram of peonidin 3,5 diglucoside

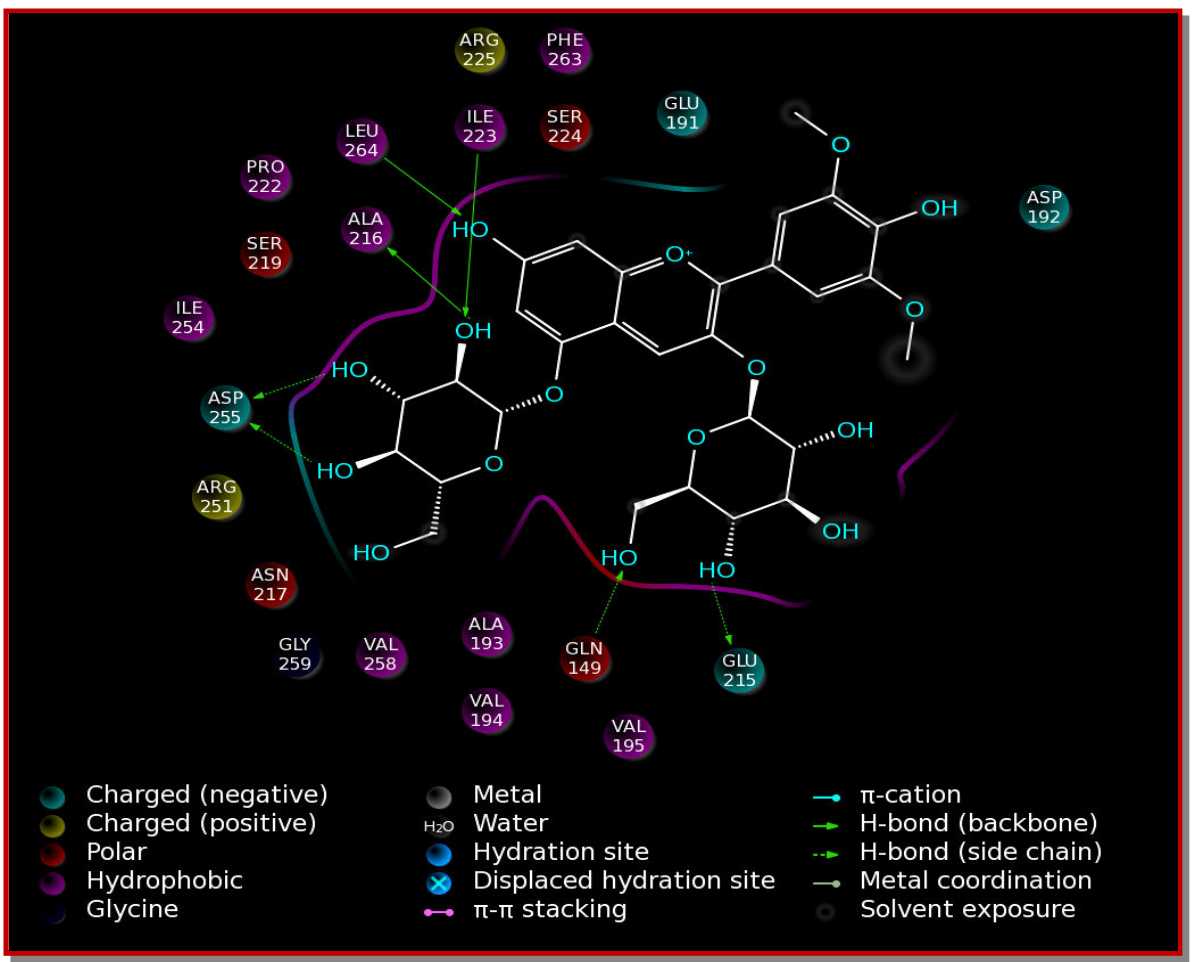

Figure 5: Interaction diagram of malvidin 3,5 diglucoside 
binding energy for the compound with lowest Gscore is depicted in Table III.

Role of cyanidin 3-glucoside in improving vision has been studied by various workers (Nakaishi et al., 2000; Tripula et al., 2009). In this study, cyanidin 3,5 diglucoside identified from the S. cumini fruit peel, strongly binds with the RP2 protein followed by other anthocyanin compounds. Precisely, anthocyanins are abundantly found in berries and their benefits to human health are widely explored (Szajdek et al., 2008). The fruit peel of S. cumini was shown to contain anthocyanins (Veigas et al., 2007).

The reliable binding free energy obtained indicates the efficacy of cyanidin, 3,5 diglucoside as a potent inhibitor for RP2. In general, regular consumption of fruits provides immense health benefits against degenerative diseases, especially in age related macular degeneration (Scharrer and Ober, 1981). Few recent studies have shown the efficacy of anthocyanins in treating inflammatory and neurogenerative diseases (Jeong et al., 2013). Recently, (Miyake et al., 2012) retinal inflammation affecting vision was treated with anthocyanins from bilberry extracts. Purified anthocyanins in clinical trials were demonstrated to improve ocular health (Lee et al., 2011). Vision loss caused by mutations in the RP2 gene has no effective treatment till date. Substantial interest in anthocyanins corresponds to their therapeutic benefits. Therefore, we docked five anthocyanins into the predicted active sites of RP2 drug target.

\section{Conclusion}

The molecular docking and binding free energy obtained in this study suggests that cyanidin 3,5 diglucoside could be a potent inhibitor in the treatment of X-Linked retinitis pigmentosa.

\section{Acknowledgement}

The authors acknowledge Mr. R. Raghu, Executive Director, Schrödinger Inc. for providing trial license of Maestro software to carry out this work.

\section{References}

Adisakwattana S, Yibchok-Anun S, Charoenlertkul P, Wongsasiripat N. Cyanidin-3-rutinoside alleviates postprandial hyperglycemia and its synergism with acarbose by inhibition of intestinal a-glucosidase. J Clin Biochem Nutr. 2011; 49: 36-41.

Ayuso C, Jose MM. Retinitis pigmentosa and allied conditions today: a paradigm of translational research. Genome Med. 2010; 2 : 34 .
Berson EL, Rosner B, Sandberg MA, Weigel-Difranco C, Willett WC. $\omega-3$ intake and visual acuity in patients with retinitis pigmentosa receiving vitamin A. Arch Ophthalmol. 2012; 130: 707-11.

Bhalla S, Joshi D, Bhullar S, Kasuga D, Park Y, Kay CN. Longterm follow-up for efficacy and safety of treatment of retinitis pigmentosa with valproic acid. Br J Ophthalmol. 2013.

Byamukama R, Kiremire BT, Andersen OM, Steigen A. Anthocyanins from fruits of Rubus Pinnatus and Rubus Rigidus. J Food Composit Anal. 2005; 18: 599-605.

DiMasi JA, Hansen RW, Grabowski HG. The price of innovation: New estimates of drug development costs. J Health Econ. 2003; 22: 151-85.

Eldridge MD, Murray CW, Auton TR, Paolini GV, Mee RP. Empirical scoring functions: the development of a fast empirical scoring function to estimate the binding affinity of ligands in receptor complexes. J Comput-Aided Mol Des. 1997; 11: 425-45.

Friesner RA, Murphy RB, Repasky MP, Frye LL, Greenwood JR, Halgren TA, Sanschagrin PC, Mainz DT. Extra precision Glide: Docking and scoring incorporating a model of hydrophobic enclosure for protein-ligand complexes. J Med Chem. 2006; 49: 6177-96.

Ghosh D, Konishi T. Anthocyanins and anthocyanin-rich extracts: Role in diabetes and eye function. Asia Pac J Clin Nutr. 2007; 16: 200-08.

Glide, version 5.8, Schrödinger, LLC, New York, NY, 2012.

Halgren TA. Identifying and characterizing binding sites and assessing druggability. J Chem Inf Model. 2009; 49: 377-89.

Hardcastle AJ, Thiselton DL, Van Maldergem L, Saha BK, Jay M, Plant C, Taylor R, Bird AC, Bhattacharya S. Mutations in the RP2 gene cause disease in $10 \%$ families with familial $X$ Linked retinitis pigmentosa in this study. Am J Hum Genet. 1999; 64: 1210-15.

Inglehearn CF. Molecular genetics of human retinal dystrophies. Eye 1998; 12: 571-79.

Jeong JW, Lee WS, Shin SC, Kim GY, Choi BT, Choi YH. Anthocyanins down-regulate lipopolysaccharide-induced inflammatory responses in BV2 microglial cells by suppressing the NF-kB and Akt/MAPKs signaling pathways. Int J Mol Sci. 2013; 14: 1502-15.

Ji HF, Li XJ, Zhang HY. Natural products and drug discovery can thousands of years of ancient medical knowledge lead us to new and powerful drug combinations in the fight against cancer and dementia? Embo Reports. 2009; 10: 194200.

Kong JM, Chia LS, Goh NK, Chia TF, Brouillard R. Analysis and biological activities of anthocyanins. Phytochemistry 2003; 64: 923-33.

Kuhnel K, Veltel S, Schlichting I, Wittinghofer A. Crystal structure of the human retinitis pigmentosa 2 protein and its interaction with Arl3. Structure 2006; 14: 367-78.

Lee J, Lee HK, Kim CY, Hong YJ, Choe CM, You TW, Seong GJ. Purified high-dose anthocyanoside oligomer administration improves nocturnal vision and clinical symptoms in 
myopia subjects. Br J Nutr. 2005; 93: 895-99.

Li T, Sandberg MA, Pawlyk BS, Rosner B, Hayes KC, Dryja TP, Berson EL. Effect of vitamin A supplementation on rhodopsin mutants threonine- $17 \rightarrow$ methionine and proline$347 \rightarrow$ serine in transgenic mice and in cell cultures. Proc Natl Acad Sci USA. 1998; 95: 11933-38.

LigPrep, version 2.5, Schrödinger, LLC, New York, NY, 2012.

Maestro, version 9.3, Schrödinger, LLC, New York, NY, 2012.

Matsumoto $\mathrm{H}$, Inaba $\mathrm{H}$, Kishi $\mathrm{M}$, Tominaga S, Hirayama $\mathrm{M}$, Tsuda T. Orally administered delphinidin 3-rutinoside and cyanidin 3-rutinoside are directly absorbed in rats and humans and appear in the blood as the intact forms. J Agric Food Chem. 2001; 49: 1546-51.

Matsunaga N, Imai S, Inokuchi Y, Shimazawa M, Yokata S, Araki Y, Hara H. Bilberry and its main constituents have neuroprotective effects against retinal neuronal damage in vitro and in vivo. Mol Nutr Food Res. 2009; 53: 869-77.

Miyake S, Takahashi N, Sasaki M, Kobayashi S, Tsubota K, Ozawa Y. Vision preservation during retinal inflammation by anthocyanin-rich bilberry extract: Cellualr and molecular mechanism. Lab Invest. 2012; 92: 102-09.

Nakaishi H, Matsumoto H, Tominaga S, Hirayama M. Effects of black currant anthocyanoside intake on dark adaptation and VDT work-induced transient refractive alteration in healthy humans. Altern Med Rev. 2000; 5: 553-62.

Pearlman DA. Evaluating the molecular mechanics PoissonBoltzmann surface area free energy method using a congeneric series of ligands to p38 MAP kinase. J Med Chem. 2005; 48: 7796-807.

Prime, version 3.1, Schrödinger, LLC, New York, NY, 2012.
Priya SSL, Devi RP, Eganathan P, Nishith ST. Structure prediction of Bestrophin for the induced-fit docking of anthocyanins, Bioinformation 2012; 8: 742-48.

Rundquist J. Low vision rehabilitation of retinitis pigmentosa. J Vis Impair Blind. 2004; 98: 718-24.

Sandberg MA, Rosner B, Weigel-DiFranco C, Berson EL. Lack of scientific rationale for use of valproic acid for retinitis pigmentosa. Br J Ophthalmol. 2011; 95: 744.

Scharrer A, Ober M. Anthocyanosides in the treatment of retinopathies. Klin Monatsbl Augenheilkd. 1981; 178: 386-89.

Szajdek A, Borowska EJ. Bioactive compounds and healthpromoting properties of berry fruits: A review. Plant Foods Hum Nutr. 2008; 63: 147-56.

Taylor CW. Anthocyanins in cardiovascular diseases. Adv Nutr. 2011; 2: 1-7.

Tiupula KC, Blaem F, Yanamala N, Klein-Seetharaman J. pHdependent interaction of rhodopsin with cyanidin-3-glucoside.2. Functional aspects. Photochem Photobiol. 2009; 85: 463-70.

Veigas JM, Narayan MS, Laxman PM, Neelwarne B. Chemical nature, stability and bioefficacies of anthocyanins from fruit peel of Syzygium cumini Skeels. Food Chem. 2007; 105: 61927.

Veltel S, Wittinghofer A. RPGR and RP2: targets for the treatment of X-linked retinitis pigmentosa? Expert Opin Ther Targets. 2009; 13: 1239-51.

Wang LS, Stoner GD. Anthocyanins and their role in cancer prevention. Cancer Lett. 2008; 269: 281-90.

Wrolstad RE. Interaction of natural colors with other ingredients. J Food Sci. 2004; 69: 419-21. 